\title{
A multidimensional phenomenal space for pain: structure, primitiveness, and utility
}

\author{
Sabrina Coninx ${ }^{1}$ (i)
}

Accepted: 6 January 2021 / Published online: 27 February 2021

(c) The Author(s) 2021

\begin{abstract}
Pain is often used as the paradigmatic example of a phenomenal kind with a phenomenal quality common and unique to its instantiations. Philosophers have intensely discussed the relation between the subjective feeling, which unites pains and distinguishes them from other experiences, and the phenomenal properties of sensory, affective, and evaluative character along which pains typically vary. At the center of this discussion is the question whether the phenomenal properties prove necessary and/or sufficient for pain. In the empirical literature, sensory, affective, and evaluative properties have played a decisive role in the investigation of psychophysical correspondence and clinical diagnostics. This paper addresses the outlined philosophical and empirical issues from a new perspective by constructing a multidimensional phenomenal space for pain. First, the paper will construe the phenomenal properties of pains in terms of a property space whose structure reflects phenomenal similarities and dissimilarities by means of spatial distance. Second, philosophical debates on necessary and sufficient properties are reconsidered in terms of whether there is a phenomenal space formed of dimensions along which all and only pains vary. It is concluded that there is no space of this kind and, thus, that pain constitutes a primitive phenomenal kind that cannot be analyzed entirely in terms of its varying phenomenal properties. Third, the paper addresses the utility of continued reference to pain and its phenomenal properties in philosophical and scientific discourses. It is argued that numerous insights into the phenomenal structure of pain can be gained that have thus far received insufficient attention.
\end{abstract}

Keywords Conceptual space · Quality space · Phenomenal quality · Quale · Necessity $\cdot$ Sufficiency

Sabrina Coninx

sabrina.coninx@rub.de

1 Institute for Philosophy II, Ruhr University Bochum, Universitätsstraße 150, GAFO 04/985, 44780 Bochum, Germany 


\section{Introduction}

Researchers from different academic disciplines, including philosophy, psychology, neuroscience, and clinical medicine, commonly consider pain in terms of subjective experience (e.g., Raja et al. 2020). Subjective experience determines the subject matter of pain research. It seems that pains in contrast to non-pains typically feel a certain way. There is something it is like to experience fracture pains, headaches, or phantom pains in contrast to itches, hunger, or grief. This common and unique feeling of pain seems to be the reason why we can identify pains as pains from an introspective perspective even though it might be difficult to say exactly what such feeling is beyond its being shared by each pain (e.g., Aydede and Fulkerson 2019; Coleman 2020; Klein 2015). This feeling of pain has been addressed in the philosophical literature under different labels-phenomenal quality, qualitative character, or quale (e.g., Byrne 2001; Clark 2005; Kripke 1981). Along these lines, one might hold that pain constitutes a phenomenal kind with a particular phenomenal quality common and unique to its instantiations (see also Corns 2018, 2020).

At the same time, we need to acknowledge that the phenomenology of pain is much more complex and variable. For example, when taking ice cubes out of the freezer, we may experience a cold and slightly unpleasant pain, while we may experience a searing and highly unpleasant pain when touching a hot plate. Hence, we may distinguish between the unique and common feeling due to which we can introspectively identify pains as pains, i.e., the phenomenal quality of pain, and the phenomenal properties, e.g., thermal character or unpleasantness, along which instantiations of pain typically vary. On the basis of this distinction, there are three issues to be addressed in this paper concerning (i) the internal phenomenal structure of pain, (ii) its primitiveness, and (iii) the resulting utility of continued reference to pain and its phenomenal properties in philosophy and sciences. These closely linked issues are explained in more detail in the following.

First, several researchers have addressed the phenomenal properties of pains (e.g., Bain 2014; Grahek 2007), but there rarely is clear indication of how these phenomenal properties should be conceptualized (Fink 2011). In psychology, dimensional accounts appear promising (e.g., Price 2000) and similar approaches are suggested in the philosophical literature (e.g., Clark 2005). However, the concrete implementation of a dimensional framework still has to be closely analyzed while taking account of the complexity and variability of pains, on the one hand, and aiming for a detailed examination of the internal phenomenal structure and organization of pains, on the other. This gap in literature seems surprising as detailed multidimensional approaches can be found in the investigation of diverse phenomena, such as perception, reasoning, or semantics (Clark 2000; Gärdenfors 2000; Osta-Vélez and Gärdenfors 2020; Young et al. 2014).

Second, based on a comprehensive conceptualization of the phenomenal properties of pain, the question arises as to the relation between the subjective feeling, which unites pains and distinguishes them from other experiences, and those properties along which instantiations of pain typically vary. There are two 
prominent options (Fink 2011). Either, pain constitutes a primitive phenomenal kind that is systematically characterized by certain phenomenal properties but not entirely analyzable in those terms. Or, pain may feel like a homogenous whole but it can in fact be entirely analyzed in the relevant manner. The latter presumes that the phenomenal properties in question can fully account for the common and unique feeling of pain. Therefore, this issue is related to debates over whether the phenomenal properties considered are necessary and/or sufficient for pain (e.g., Bain 2014; Corns 2014a; Grahek 2007; Klein 2015). Further, this allows us to address the more general question of how to account for a phenomenal quality that is supposed to be common and unique to pains, despite the complexity and variability of their phenomenal properties (e.g., Aydede and Fulkerson 2019; Coleman 2020; Clark 2005).

Third, the phenomenal kind status of pain is a core topic in philosophical debates, particularly as pain is often used as the paradigmatic example for an experience with a particular phenomenal quality. If pain is a primitive phenomenal kind, this seems to renders the common and unique feeling of pain somehow mysterious. Moreover, the phenomenology of pain plays a decisive role in different areas of scientific research. For example, the phenomenal properties along which pains vary are systematized for diagnostic purposes and linked to neural activity patterns or medical conditions (e.g., Dubuisson and Melzack 1976; Melzack and Katz 2013; Price and Aydede 2005; Roy and Wager 2017). Based on the phenomenal structure and organization of pains, different approaches may seem more promising. If pain is a primitive phenomenal kind, these scientific endeavors may appear pointless insofar as they do not concern the intrinsic nature of pain (Fink 2011).

In the course of this paper, I will systematize the phenomenal properties of pains in terms of a multidimensional phenomenal space relying on previous work in the development of multidimensional property spaces - conceptual, perceptual, or qualitative-and their application to pain (Clark 2005; Coleman 2020; Coninx 2020a; Corns 2014a; Gärdenfors 2000). Thus, I will use this general approach to reconsider existing debates in pain research. This allows me to connect philosophical considerations with corresponding empirical results in a unified framework. Based on the previously introduced issues, such project relies on three consecutive steps.

First, by identifying and constructing those dimensions which account for the phenomenal differences across pains, we can capture and display the diverse facets of the phenomenology of pain. Thus, this provides a consistent implementation of a dimensional approach to pain in due consideration of the complexity and variability of its instantiations. The resulting topology may help us better understand the phenomenology of pain by revealing its internal organization in terms of spatial distance. Second, the multidimensional framework provides refined criteria and interesting insights which respect to the debate on the necessity and sufficiency of phenomenal properties. Most importantly, we shall see that pain proves primitive as we are unable to map a multidimensional phenomenal space in terms of which we can entirely analyze the common and unique feeling of pain. Third, the multidimensional framework allows us to shed light on the phenomenal structure of pain which proves beneficial for various scientific discourses, independent of whether pain constitutes a primitive phenomenal kind or not. At the same time, this general approach 
might also help us better understand the remaining philosophical challenges that stem from the assumption of a phenomenal quality common and unique to pains.

The overall aim of this paper is to construct a multidimensional phenomenal space for pain and to prove its usefulness in pain research. The paper proceeds as follows. In $\S 2$, the general idea of property spaces is introduced and possible dimensions of a phenomenal space for pain are identified. The mapping of such a multidimensional space allows us to systematize the variation across pains along clearly defined principles. In $\S 3$, I show how a multidimensional approach enables us to reconsider the arguments that are commonly put forward in the debate on the necessity and sufficiency of phenomenal properties. This leads us to the conclusion that pain is a primitive phenomenal kind. In $\$ 4$, I argue that the framework of geometric spaces nonetheless provides a starting point for understanding how an investigation of the complexity and variability of pain can be fruitful. The construction of a multidimensional phenomenal space allows for key insights concerning both the scientific investigation of pain as well as its philosophical understanding that have largely been overlooked in the literature thus far. $\$ 5$ summarizes the conclusions of the paper.

\section{Phenomenal structure}

As one of the most famous examples of multidimensional property spaces, conceptual spaces are considered mathematical entities constituted by a combination of quality dimensions within a geometric space representing properties that objects can possess to different degrees (Gärdenfors 2000). These quality dimensions reflect the different manners in which stimuli can appear and be judged as similar or different. Depending on the degree to which they possess the properties represented by the relevant dimension, objects can be mapped onto coordinate points in the multidimensional space. The geometric structure of this space enables us to use spatial distance as a measure of similarity with respect to the properties that the dimensions model. Paradigmatic examples of similarity spaces are the color space, gustatory space, or various auditory spaces, with dimensions such as brightness, sweetness, or pitch.

The aim of this paper is to use this general framework of a multidimensional property space in order to study pain and its phenomenal properties. In a first step, we shall therefore map the phenomenal properties along which pains vary in terms of dimensions, which in combination form a geometrically defined phenomenal space. The main motivation to construct such a phenomenal space is to account for the complex phenomenology of pain, systematize the phenomenal variation across pains, and investigate the corresponding topological structure. ${ }^{1}$

This project is based on the assumption that pain constitutes a phenomenal kind. Besides their complexity and variability in phenomenal properties, there seems to

\footnotetext{
1 This paper does not concern the notion "pain" and the construction of a conceptual space that represents the different manners in which it is used (e.g., Borg et al. 2020). The focus is not to better understand the composition and acquisition of concepts, but to develop an approach to the phenomenal complexity and variability of pain.
} 
be a phenomenal quality common and unique to pains that cannot be further distinguished (Clark 2005). Thus, this phenomenal quality cannot be identified with any of the dimensions of a possible phenomenal space that expresses phenomenal variation (Coninx 2020a). If pain is a phenomenal kind, then there should be no systematic variation: a person experiences pain or not. However, we might outline an independent space or domain representing the phenomenal similarities and dissimilarities of pains. According to the general framework of property spaces (Gärdenfors 2000), such a space or domain is a set of multiple integral dimensions, such as the color space, that represent a particular set of entities, such as colors. Integral dimensions are those where an object necessarily possesses a value given that it possesses a value on another dimension of the multidimensional property space. Paradigmatically, this seems to be the case when considering the dimensions of hue, brightness, and saturation in the color space.

Colors do not differ with respect to the property of being a color. However, a color space can represent the variation of colors concerning hue, saturation, and brightness that, in combination, constitute a color space (see also Coleman 2020). The aim is to construct a space or domain for pain by analogy to a color space or color domain, which represents the particular set of experiences introspectively qualified as pains and models their phenomenal complexity and variability. Thus, when speaking about the phenomenal properties along which pains vary, I do so by analogy to properties such as hue, saturation, and brightness (in contrast to, for example, Gärdenfors and Warglien (2012)). The philosophical and scientific relevance of these considerations will be addressed in more detail in the following sections. The remainder of this section focuses on the construction of a plausible phenomenal space that inhabits paradigmatic cases of pain.

Before addressing this task, I note that it does not assume that pain constitutes a sense modality similar to vision, taste, or audition. Gärdenfors (2000) primarily considers corresponding spaces because they seem to have close connections with the processing of particular sensory signals of which visual, gustatory, or auditory perceptions are supposed to be interpretations. ${ }^{2}$ It is still controversial whether or not pain constitutes such form of perception (Coninx 2021; Corns 2014a; Klein 2015). This paper aims to remain neutral on this issue. Whether an isomorphism between the phenomenal properties of pain and certain physical properties, accessible via our sensory apparatus, is possible or not is an independent question (see §4). I use the term "phenomenal" to refer to properties of subjective experiences and not mind-external objects or their appearance and, thus, no difference is made between phenomenal and quality spaces (Clark 2000). Strictly speaking, pains are therefore aligned with color experiences.

In the field of diagnostics, we can find categorizations of the phenomenal properties of pain, for example, in the form of the McGill Pain Questionnaire (Melzack 2005). These taxonomies address the complexity of the phenomenology of pain but often lack a clear-cut definition of the relevant phenomenal properties that avoids

\footnotetext{
${ }^{2}$ Interestingly, Gärdenfors et al. (2018) also speak of a "pain space" that is supposed to relate in a particular manner to sensory stimuli. Unfortunately, they do not elaborate on how to exactly model this space.
} 
overlaps or potential mutual exclusions among these properties. In addition, these taxonomies often rely on the selection of adjectives that denote distinct qualities and, thus, make comparisons between pains and translations across linguistic boundaries difficult (e.g., Wierzbicka 2012). Constructing a multidimensional phenomenal space for pain provides an opportunity to do better, since overlaps and mutual exclusions are prevented by the principles, explained below, that guide the construction of the property space. Moreover, the dimensions of the envisaged phenomenal space express differences in magnitude making the phenomenal properties quantifiable and their instantiations comparable. Different sections of the dimensions can be associated with certain adjectives, like those used in the McGill Pain Questionnaire, but these may vary across linguistic communities and need not be translatable oneto-one. Examples of such associations are given below.

The construction of a phenomenal space for pain should be guided by the following three principles (Clark 2005; Gärdenfors and Warglien 2012). First, the coordinate points on a dimension are in general supposed to represent the different degrees to which objects possess a property. Thus, different values on a dimension should represent minimal subjectively experienceable differences in the phenomenal properties considered. For present purposes, we may assume that these dimensions have a simple Euclidean metric and that the smaller the spatial distance between the values that pains instantiate on a dimension the more similar they are. Second, it should be possible, at least in principle, that a pain possesses all the properties mapped in the envisaged phenomenal space to different degrees. That is, the dimensions that form the geometric space should be able to constitute integral dimensions as defined above. This implies that the dimensions that we construct must not be mutually exclusive. Third, for the sake of economy, the fewer dimensions the better. If we can account for a certain phenomenal property in terms of the (combined) values that pains instantiate on existing dimensions, we should not add another one. However, if the values that pains instantiate along different dimensions can vary independently, these dimensions should be considered independently. This does not exclude the possibility that the properties that are modeled in the geometric space often co-vary.

To begin the construction of a phenomenal space, we consider those phenomenal properties along which pains typically vary and systematically outline the dimensions that can be plausibly used to model this variation. This is to prevent the introduction of arbitrary dimensions. For this purpose, we may consider standardized inquiries that list and categorize adjectives commonly used to qualify the varying aspects of pains (e.g., Melzack 2005), as well as philosophical and psychological debates concerning characteristic phenomenal properties of pains (e.g., Bain 2014; Corns 2014a, b; Cutter 2017; Fink 2011; Grahek 2007; Klein 2015; Price 2000). As we shall see, both sources indicate a rough systematization of sensory, affective, and evaluative properties, which will be considered in the following in accordance with the previously introduced principles. ${ }^{3}$

\footnotetext{
3 The following discussion of the sensory, affective, and evaluative dimensions develops, refines, and adjusts an approach first introduced in Coninx (2020a) in due consideration of recent work on pain and the relevant principles of constructing property spaces (e.g., Clark 2005; Coleman 2020; Corns 2014a; Gärdenfors 2000).
} 
First, different pains can be specified along three sensory dimensions of temperature, force, and saturation. Before outlining these dimensions, two disclaimers are in order. First, pains differ phenomenologically with respect to their felt bodily location: pains can be experienced in all different parts of the body, including tiny sections of the skin or whole organ complexes. This phenomenal property is itself a spatial-topological property that cannot be quantified. For the sake of simplicity, I only focus on phenomenal properties that pains might instantiate to different degrees and on dimensions that stand for variation in magnitude. ${ }^{4}$ Second, pains are typically considered to vary with respect to their intensity. The problem with intensity ratings is that they might not express the magnitude of an independent phenomenal property. For example, imagine feeling a mildly warm pain in contrast to a searing pain. These pains differ in terms of the intensity of their thermal character, but there might be no additional property that needs to be captured by an independent dimension of intensity. On the assumption that this is correct, our principle of economic parsimony comes into play: we do not need a separate dimension for pain intensity but we can attribute the corresponding ratings common to the pain literature to changes in values of other dimensions, individually or perhaps in combination.

After these two disclaimers, we shall now address the sensory dimensions that are considered in the construction of our phenomenal space. The McGill Pain Questionnaire (Melzack 2005) suggests categorizing the remaining properties along seven sub-groups related to temperature (e.g., "warm", "burning"), puncture pressure (e.g., "boring", "lancinating"), incisive pressure (e.g., "cutting", "lacerating"), constrictive pressure (e.g., "cramping", "crushing"), traction pressure (e.g., "drawing", "wrenching"), brightness (e.g., "stinging", "sharp"), or dullness (e.g., "sore", "aching"). The miscellaneous category includes further adjectives related to some of the previously introduced groups (e.g., "cold", "piercing"). In the philosophical literature, sensory properties of different kinds are often mentioned but they are rarely systematized (e.g., Clark 2005; Cutter 2017; Klein 2015). They are most plausibly construed in terms of three dimensions, satisfying the previously introduced principles. A one-dimensional thermal scale accounts for phenomenal properties ranging from the subjective experience of very low to very high temperature with a zero point in the middle. Another one-dimensional scale refers to force, ranging from a strong inward pressure to a strong pulling outwards as opposite extremes. A final one-dimensional scale accounts for the saturation, or depth, of pains, ranging from values associated with adjectives of brightness to values associated with adjectives of dullness with a zero point in the middle.

These three dimensions allow us to account for different adjectives that subjects might use to describe the sensory aspects of their pains, especially when alterations in bodily location are also integrated. Consider the following examples for illustration. A pain described as "piercing" might be understood as being rather pressing than pulling, more or less neutral in terms of temperature, bright in terms of

\footnotetext{
${ }^{4}$ Please note that pains are typically experienced as located in a certain part of the body, but this is not the same as occupying a location in the phenomenal space constructed by dimensions that model quantifiable phenomenal properties.
} 
saturation, and most likely felt in a restricted small part of the body. Differences between adjectives that denote pains of inward pushing force (e.g. "lancinating", "crushing", "cutting") can be explained by taking into account the strength of pressure, the area of the body over which it is distributed, and the saturation of the respective experience. Fluctuations in location and magnitude of temperature, pressure, or saturation might account for qualifications, such as "radiating", "flickering", or "jumping".

Second, we shall address the affective dimension. In this regard, the focus of debate in philosophy and psychology is primarily on the negative valence of pain (Bain 2014; Corns 2014a; Grahek 2007). This property can be construed in terms of a dimension that grows with the magnitude of felt unpleasantness (Price 2000). Some authors suggest a dimension with two mutually exclusive extremes modeling magnitudes of negative and positive valence (e.g., Borg et al. 2020). I will model the affective dimension only in terms of unpleasantness as the valence of pain is negative in paradigmatic cases. Moreover, the central question of philosophical discussions is not so much whether pains can have a positive valence in individual cases, but whether there are cases in which it is not negative (e.g., Clark 2005; Coleman 2020; Corns 2014a). ${ }^{5}$

The final property to consider is the evaluative property. In the McGill Pain Questionnaire, evaluative variations are associated with adjectives such as "distracting", "agonizing", "savage", and "excruciating" (Melzack 2005). The evaluative property is related to the meaningful evaluation of a subject's situation in the context of past and present experiences (Melzack and Wall 1982). The evaluative property is to be understood as a secondary pain affect (Price 2000) or secondary unpleasantness (Fields 1999). In contrast to moment-by-moment (primary) unpleasantness, it concerns the felt disruption of long-term plans and goals as well as the negative implications of pain for the subject's overall well-being. The evaluative property seems particularly relevant for characterizing the existential changes that pains can bring about: pain can shift a person's entire focus so that, in extreme cases, it appears to be the only thing that exists for the subject while the rest of the world vanishes.

This evaluative aspect has been often neglected in the philosophical debate. At best, it seems reflected in the literature on suffering, i.e. a phenomenon that is commonly distinguished from the (primary) unpleasantness of pain and interpreted in experiental terms as the negative affective construal of a subject's overall situation (Kauppinen 2020) or the holistically unpleasant disruption of one's mental life (McClelland 2020). Parallel to the moment-by-moment unpleasantness (Price

\footnotetext{
5 The affective aspect of pain is usually associated with a motivational aspect concerning some sort of felt aversion. While it seems to be commonly accepted that sensory and affective aspects can be independently manipulated in certain experimental settings (e.g., Price 2000), it is subject of an ongoing debate whether this also applies to affective and motivational aspects (Bain 2013, 2014; Corns 2014b; Klein 2015). This empirical debate is relevant for deciding whether our principle of economic parsimony applies. For the sake of simplicity, we shall focus here merely on the affective dimension. However, the following considerations are in principle compatible with the position that there are two separate dimensions indicating the degrees of affective valence and motivational force while every value on one dimension is compatible with any value on the other dimension.
} 
2000), we might account for the evaluative property by means of a one-dimensional scale that increases with the degree of subjective experience of restriction, debilitation, or disruption in the given pain.

We have thus arrived at a phenomenal space with five dimensions representing values with increasing magnitude. There are three sensory dimensions, temperature, force, and saturation, with two opposite extremes. The remaining two are affective and evaluative dimensions. Each of which extend into a negative or restricting range. The resulting multidimensional space lays the foundation for exploring the topology of the phenomenal complexity and variability of pains (e.g., Melzack and Katz 2013). The spatial distance of the corresponding coordination points represents their similarity or dissimilarity with respect to the phenomenal properties modeled by the dimensions mentioned above.

\section{Primitiveness}

\subsection{Phenomenal quality \& phenomenal space}

We began our considerations with the assumption that pain constitutes a phenomenal kind whose instantiations vary along particular sensory, affective, and evaluative properties. On that basis, the question arises how the unique and common feeling of pain relates to these quantifiable phenomenal properties. At first glance, it seems that there are two possible options concerning the primitiveness of pain (Fink 2011). According to the first option pain constitutes a primitive phenomenal kind. This means that the phenomenal quality of pain cannot be entirely analyzed in terms of the considered phenomenal properties or their combination. Pains may vary across the corresponding dimensions, but their being experienced as pains does not depend on them doing so. The unique and common feeling of pain cannot be reduced to or broken down into sensory, affective, and evaluative properties. According to the second option, we can entirely analyze pain in terms of the phenomenal properties previously introduced, even if such analysis is not possible from an introspective perspective. According to this view, there might be nothing primitive about pain.

One way to investigate which of these options is more plausible is in terms of the multidimensional phenomenal space that we have just outlined. Thus, the relevant question is how the subjective experience, which unites pains and distinguishes them from other phenomena, relates to the phenomenal space that models variation across pains. Phenomenal spaces, or quality spaces, have played a substantial role in the philosophy of pain, first and foremost in the discussion of the question whether pain, or painfulness, constitutes a distinct quality. The beginning of this debate lies in the work of Saul A. Kripke (1981) who argues for a phenomenal quality that allows us to pick out all and only pains. Kripke's statement has been commonly interpreted in essentialist terms and critically discussed in philosophy, among others, by Austen Clark (2005) and Jennifer Corns (2014a).

Clark (2005) reasons as follows: a particular quale is a fully determined quality that does not possess any determinable components. It is impossible to discriminate 
between different instantiations of a quale with regard to any particular phenomenal aspect. According to Clark, a quale must therefore correspond to one concrete coordinate point in a quality space. As we have seen, pain has various phenomenal properties, but these are properties with regard to which pains vary (Grahek 2007). Some pains feel warm, pressing, and dull; others feel cold, wrenching, and sharp. Some pains are very unpleasant; others are much less unpleasant. Some pains have values on the evaluative dimension best characterized as "distracting"; others have values best characterized as "savage". Thus, pain cannot be identical to any value on any dimension that might construct a phenomenal space for pain. As a direct consequence, pain cannot be identified with a distinct coordinate point within such a geometric space either.

An alternative interpretation of the claim that pain can be identified with a particular phenomenal quality can be found in the work of Jennifer Corns:

(...) perhaps the distinctive pain quale is actually any quale that is a member of a pain quality space. This view allows token pains to qualitatively vary (...) while nonetheless characterizing the type, pain, by a distinct family of qualitative content. (...) Experiences of red and blue, despite lacking any common qualitative content, can be arranged into a color quality space by their similarities and differences across three dimensions: hue, saturation, and brightness. (...) Red and blue sensations are both color sensations that can be characterized in this way; i.e., they are both color sensations even if they vary in hue, saturation and luminance, just in virtue of being characterizable by hue, saturation, and luminance. Similarly, on the proposed interpretation, no one point within the pain quality space is essential to a token mental state being a pain. Rather, what is essential is that the mental state can be characterized as lying somewhere within the pain quality space. (Corns 2014a, p.364).

Corns considers the outlined proposal as an unfortunate interpretation of Kripke's view. While this interpretation may fail to do justice to Kripke's original point, it might be useful for present purposes.

It seems obvious that pain can be identified neither with any of the dimensions previously introduced nor with any value of these dimensions nor with any particular coordinate point in the geometric space that the dimensions form. However, in line with the alternative interpretation just outlined, one could argue that pains are experienced as pains simply in virtue of instantiating a coordinate point within a particular quality space (Coleman 2020; Grahek 2007). Thus, we may think of the phenomenal quality of pain as a determinable of which each individual case of pain is a determinate independent of the variation in the phenomenal properties they respectively instantiate (Funkhouser 2006). This allows us to address the heterogeneity problem, i.e. the problem of identifying a phenomenal quality common to a phenomenon despite the possibly striking differences between its instantiations (e.g., Aydede and Fulkerson 2019; Coleman 2020). Consequently, we may avoid the identification of pain as a primitive phenomenal kind as we can fully analyze the common and unique feeling of pain in terms of the combination of quantifiable phenomenal properties along which its instantiations vary. 
The claim that pains are experienced pains in virtue of instantiating coordinate points within a particular phenomenal space seems plausible only if the multidimensional space satisfies the criteria of necessity and sufficiency. If we cannot identify a combination of phenomenal properties that is necessary as well as sufficient for pain, then it seems that pain cannot be entirely analyzed in reference to the multidimensional space that models the variation along these phenomenal properties. Either we would fail to provide a domain with integral dimensions onto which all pains could be mapped. Or, some non-pains would occupy a location within the respective domain. In each case, instantiations of pain could not be identified as such in virtue of instantiating a coordinate point within the phenomenal space. In contrast, if we can identify phenomenal properties that are necessary and sufficient for pain, then it seems possible to analyze the phenomenal quality of pain entirely in terms of the corresponding multidimensional property space.

In the following subsections, I will test the phenomenal space outlined in $\S 2$ with respect to the criteria of necessity and sufficiency. It is important to keep in mind that only two alternatives concerning the primitiveness of pain are considered here and that the exclusion of one alternative is considered as confirmation of the other. Thus, postulating that pain is a primitive phenomenal kind is defined as the impossibility to analyze the phenomenal quality of pain in terms of the previously introduced phenomenal space. In this sense, I will conclude that pain is a primitive phenomenal kind.

\subsection{Necessity}

In this section, I will consider whether the previously mapped phenomenal space constitutes a domain with integral dimensions and, thus, whether pains that instantiate a value on one of the dimensions necessarily instantiate values on the others. This is closely related to the philosophical debate over whether certain phenomenal properties are necessary aspects of pain or merely typical characteristics which may be absent in some fringe cases. First and foremost, we need to characterize the criterion along which we consider the relation between pain and a certain phenomenal property necessary. The commonly applied criterion to decide this issue seems to be the following (e.g., Fink 2011):

Criterion of Necessity: If a subject stops experiencing pain when a certain phenomenal property $\mathrm{P}$ is blocked (all other things being equal), then $\mathrm{P}$ is necessary for pain to emerge.

Put another way, if we can identify a case in which the respective phenomenal property $\mathrm{P}$ is absent while the subject continues to experience pain, then this property $\mathrm{P}$ is not necessary. This criterion needs further explanation. For instance, what does it mean for a phenomenal property to be blocked or absent? It seems implausible to request that all pains instantiate a phenomenal property to the same degree. Nor can we claim that a phenomenal property is blocked because a pain fails to instantiate a particular value on the respective dimension (see §3.1). 
Alternatively, one might assume that a phenomenal property is blocked when the value that a pain instantiates on the respective dimension is identical to the origin of the dimension. That is, a pain which scores zero on a dimension would be without the corresponding property. This approach is flawed. An individual case of pain may score zero on a certain dimension, but this dimension still characterizes a person's experience of pain. A pain that is felt as being of body temperature still seems to have a thermal character, even though it scores zero on the corresponding dimension as it is subjectively experienced as neither warm nor cold. Similarly, some shades of gray are characterized by brightness, even though they are located at the zero point of this dimension. At the same time, it is almost impossible to subjectively distinguish whether a particular pain scores zero or not. For example, in the case of very mild pains. Consequently, we might end up with opposite intuitions about whether particular instantiations of pain score zero or almost zero. That is, over whether they are of vanishingly small negative valence or of no negative valence at all. Disputes about whether mild pains are really unpleasant or not do not seem to be very fruitful.

Does this mean that we have to give up the idea of a criterion that allows us to constructively discuss whether a phenomenal property is absent or not? No. We might plausibly consider a phenomenal property blocked when pains instantiate no value on the corresponding dimension. The framework of multidimensional spaces allows us to refine the criterion of necessity as follows:

Dimensional Criterion of Necessity: If a subject stops experiencing pain when a certain phenomenal property $\mathrm{P}$ is blocked (all other things being equal), then $\mathrm{P}$ is necessary for pain to emerge. We can speak of a phenomenal property $\mathrm{P}$ being blocked, when a pain instantiates no value on the respective dimension.

Based on this dimensional criterion of necessity, we may avoid the issues previously outlined. We can accept that a pain might occupy any value on a dimension, irrespective of whether the location of such value is identical to the zero point, the maximum, or equivalent to any other magnitude in between. Thus, we also avoid discussing whether a particular pain scores exactly zero on a certain dimension or not. At the same time, we can consider a phenomenal property being blocked or absent when a pain occupies no value on the respective dimension.

In more practical terms, it may still seem difficult to distinguish between a case where an instantiation of pain takes on a value of zero and a case where an instantiation of pain has no value, especially as pains may subjectively appear as homogenous units. However, we may distinguish between the two described cases by means of various manipulations, e.g., by significantly increasing an experimental stimulus so that the experienced value of the phenomenal property would, under normal conditions, rise well above zero. This should not happen, however, if the pain occupies no value on the corresponding dimension, for example, due to a subject's general inability to experience pains of this kind (Coninx 2020a). Therefore, neurophenomenological case studies that allegedly show that certain phenomenal properties are absent are of particular relevance (Fink 2011).

Many philosophers seem to assume that at least some of the phenomenal properties of pain can be blocked under particular circumstances (e.g., Coleman 2020; 
Corns 2014a; Grahek 2007; Hall 1989; Klein 2015). Note that the point of these authors is not that all phenomenal properties are simultaneously blocked, but that groups of sensory, affective, and evaluative properties are in certain circumstances allegedly absent, typically in the presence of the other phenomenal properties. My argumentative aim is not to finally prove that the considered phenomenal properties are necessary for pain. Instead my aim is to show how existing arguments in the philosophical debate can and must be reassessed in the context of a multidimensional approach. In doing so, we might find that the evidence that allegedly proves the existence of pain cases in absence of the respective phenomenal property is rather weak (see also Coninx 2020a; Park 2019). If we employ a dimensional reading of the criterion of necessity, there are no clear-cut cases that violate such criterion. For example, the adjective "unpleasant" is commonly used to indicate a value on the affective dimension of rather moderate magnitude while the usage of said adjective seems inappropriate in the context of rather mild or severe pains (Aydede 2019). That means, if someone claims that some pains are not unpleasant, we should be careful to take into account that "unpleasant" might not denote the more general property of negative affective valence that comes in various degrees.

First, few researchers have claimed that pain can occur with sensory properties being blocked (Grahek 2007). If anything, cases of brain injuries or neural disorders are considered to be cases in which people cannot specify what their pain feels like. For example, concerned patients might reject adjectives from a given word list, including "warm", "cold", or "burning", as being inaccurate (e.g., Ploner, Freund and Schnitzler 1999). This observation alone allows for several interpretations. It is possible that some pains encompass a rather hazy and maybe strongly fluctuating value with respect to a dimension and, thus, can hardly be associated with any particular adjective. However, the inability to make a precise statement about the thermal character of a pain does not necessarily imply that the respective phenomenal property is blocked. We need to carefully distinguish whether a patient's statement concerns the adjectives that are associated with different sections of a dimension or their combinations, or whether it refers to the phenomenal property that is modelled by the dimension itself.

Second, many different cases have been discussed to show that the affective property can be blocked while subjects continue to experience pain (Corns 2014a; Fink 2011; Hall 1989). In the course of debate, most cases have turned out to provide little empirical evidence with pain asymbolia syndrome remaining the only apparent counter-example (Aydede 2005; Bain 2013; Grahek 2007). In the course of this disorder, subjects report themselves to experience pain in a certain body part while differentiating its sensory properties. At the same time, they do not describe their pains as unpleasant (e.g., Berthier, Starkstein, and Leiguarda 1988). Often neglected in the debate, asymbolic patients qualify their experiences as intolerable if the stimulus intensity is further increased. Thus, these patients have higher pain tolerance and endurance, but the described symptoms do not justify the claim that the affective property is indeed absent (Coninx 2020a; Park 2019). In contrast, pain asymbolia might merely involve a reduction in the magnitude of negative affective valence that slowly increases with stimulus intensity. Again, if such patients claim, for example, 
that their pains are not unpleasant, we should be careful to take into account that the adjective "unpleasant" might not denote the general property of affective valence.

Third, we shall consider the evaluative property. Here, I concentrate on cases in which the evaluative property is allegedly blocked and in which the influence of morphine, barbiturates, and other substances proves particularly relevant (Barber 1959; Hall 1989; Keats and Beecher 1950; Melzack 1961). Due to the administration of such substances, patients report that they no longer worry about their pain and, as such, it may seem that the "secondary affect" that I have identified with the evaluative property is absent (Grahek 2007). In these cases, the fear, anger, and frustration of subjects is altered and they become indifferent with respect to their pain. However, just as in the case of pain asymbolia, we must carefully distinguish between the blocking of a phenomenal property and its diminishment. We find some authors pointing to a minimization of the evaluative property due to, for example, morphine (Melzack 1961), while others point to complete elimination (Barber 1959). Similarly, when scientists speak about corresponding pain relief, this does not necessarily indicate that the subject is not bothered by the pain but that the magnitude of experienced restrictions on one's life and well-being are significantly reduced. This is further supported by the fact that the relief value of morphine comes in different degrees and changes over time (e.g., Paice et al. 1996).

It is perhaps the most controversial to argue for the very possibility that the evaluative property might prove necessary for pain and a more detailed examination of other apparent counterexamples is surely required. However, the dimensional criterion could help us to distinguish between cases in which the evaluative property is absent and cases in which it is only diminished. For example, we should be aware that the ascription of "suffering" might be reserved for experiences above a certain evaluative threshold (e.g., McClelland 2020). In conclusion, it seems at least possible that the multidimensional space constitutes a domain of integral dimensions.

\subsection{Sufficiency}

In this subsection, I will consider whether the phenomenal space of sensory, affective, and evaluative dimensions allows us to distinctively characterize pains in the same manner as the property space of hue, saturation, and brightness allows us to distinctively characterize colors. We are thus testing our phenomenal space with respect to the criterion of sufficiency. In the general philosophical debate, the criterion of sufficiency is typically understood in the following manner (e.g., Corns 2014a; Fields 1999; Grahek 2007):

Criterion of Sufficiency: If we can identify a case in which the phenomenal property $\mathrm{P}$ is instantiated but the subject does not experience pain, then the phenomenal property $\mathrm{P}$ is not sufficient.

The most prominent cases discussed in the philosophical debate are those in which an experience other than pain instantiates the same phenomenal property. It should be pointed out that this criterion of sufficiency also requires a closer look. When we speak of the instantiation of the same property, we of course do not mean 
that pain and non-pain cases must instantiate a property, such as negative valence, to the exact same degree. By contrast, it is relevant to discuss whether pain and nonpain cases vary along the same dimensions, independent of the exact values they instantiate. As above, we can refine the criterion in dimensional terms:

Dimensional Criterion of Sufficiency: If we can identify a case in which the phenomenal property $\mathrm{P}$ is instantiated but the subject does not experience pain, then the phenomenal property $\mathrm{P}$ is not sufficient. This is the case when an experience other than pain instantiates any value along the dimension modeling the respective phenomenal property $\mathrm{P}$.

In applying this dimensional criterion of sufficiency, we see that it is in fact violated. Other sensations than pains are experienced as burning, cold, cramping, drawing, stinging, or dull (e.g., Corns 2020; Grahek 2007). Multiple experiences, related to itch, fear, or heartbreak, can be experienced as unpleasant without being experienced as pains (e.g., Fink 2011; Klein 2015). Various experiences may involve the subjective experience of situations as meaningful disruptions of one's life, such as grief over the end of a relationship, anger about a failed job application, or the hunger of starving (e.g., Kauppinen 2020). Moreover, the multidimensional phenomenal space itself is not sufficient as experiences with a different phenomenal quality can be mapped onto coordinate points in this very space (e.g., Coninx 2020a; Fields 1999). At least other homeostatic sensations, such as hunger or itches, allow for sensory discrimination, are unpleasant to different degrees, and vary with respect to the evaluative dimension. For instance, itches are typically unpleasant and can be experienced as slightly warm, pressing, and stinging. They can be "distracting" and even turn "savage" when persisting. Thus, the subjective difference between pains and itches cannot be grounded in differences concerning the dimensions along which they vary.

At this point, one might object that pain, itches, hunger, and the like cannot be located in the same multidimensional space because they are not directly comparable with respect to the values that they instantiate on the dimensions of the phenomenal space. This objection is based on the intuition that we cannot plausibly claim that, for instance, a pain is twice as negative with respect to its affective valence as an itch. Claiming that all these phenomena are located in the same phenomenal space means that they are directly comparable with respect to the magnitude of their phenomenal properties. However, one might argue that pain and these other sensations are of completely different character and comparisons of this kind are not possible. Thus, my assumption that instantiations of these different phenomena occupy coordinate points in the same phenomenal space would be false.

I acknowledge that we cannot compare, for instance, the phenomenal properties of pains with those of color experiences. Claiming that a headache is three times as intense as an experience of red seems meaningless. This corresponds to the fact that colors and pains are not mapped onto the same space. However, I do not see any compelling reason why pains cannot be compared to itches with respect to the degrees of saturation or the magnitude of their unpleasantness. The phenomenal properties along which pains and non-pains vary are likely felt by the subject to be part of an integrated homogenous experience. This makes it difficult, from 
a first-person perspective, to extract a particular phenomenal property that can be compared to the properties extracted from other experiences. This would make even the comparison of two different kinds of pains, such as headaches and labor pains, a difficult task. Nonetheless, the analysis of how subjects typically experience pain, hunger, or itches indicates that these phenomena instantiate values along the dimensions of the same phenomenal space.

In response, one might argue that, although pain cannot be identified with a certain multidimensional phenomenal space, there might be a convex region located within a more general domain that proves necessary and sufficient. In the framework of conceptual spaces, colors, such as red, green, or blue are mapped onto the multidimensional color space as convex regions (Gärdenfors 2000). A region is considered convex when all coordinate points between clearly defined values on the dimensions of the space are occupied only by instantiations of the same kind. If this criterion is satisfied by pain, we might identify pains as those experiences occupying a point in a distinctively defined region even though the domain with its integral dimensions is not sufficient to distinguish pains from sensations of a different phenomenal quality. The decisive question is whether we have good reason to propose such a convex region.

Given their phenomenal variation, instantiations of pain occupy coordinate points at almost every part of the property space and take diverse values on all dimensions (e.g., Grahek 2007; Melzack and Katz 2013). This means that the convex pain region must be almost identical to the entire geometric space. However, this seems implausible given that, for example, hunger and stomachache, or itching and the pain of superficial injury, do not seem to differ so substantially from each other that we can exclude an instantiation of one of these phenomena being located in the area between coordinate points that are occupied by instantiations of pain (Coninx 2020a; Roy and Wager 2017). Consequently, it seems implausible that all coordinate points between the coordinate points occupied by the most contrasting pains are occupied only by pains and not by instantiations of other kinds of sensations, as required by Gärdenfors' (2000) criterion.

To summarize, assuming that pain is a phenomenal kind, the subjective feeling which allows us to identify pains as pains cannot be entirely analyzed in terms of a multidimensional phenomenal space or a distinct region within such space. Consequently, pain seems to be in fact a primitive phenomenal kind. Interestingly, the decisive problem is not a problem of heterogeneity but of demarcation.

\section{Scientific utility \& philosophical implications}

The previous considerations lead us to the conclusion that pain is a primitive phenomenal kind. Sensory, affective, and evaluative properties cannot fully account for the feeling common and unique to pains. Thus, it may seem that the phenomenal quality of pain must rather be identified with something that remains when we subtract the quantifiable phenomenal properties along which the instantiations of pain vary (Corns 2020). For example, one might assume that pain is best identified with a fundamental "ouch" which is more primal than sensory discrimination or affective 
valence (e.g., Davis et al. 2015). Consequently, it may seem that investigations of the phenomenal properties of pain fail to provide any scientifically useful insights as they do not characterize the actual nature of pain (Fink 2011). In contrast, I do not think that we need to abandon the phenomenal properties of pain as useful targets of scientific investigations. The study of the multidimensional phenomenal space provides numerous insights that are of use for different scientific purposes.

Sensory, affective, and evaluative properties systematically characterize the experience of pain, even if they are not sufficient to account for the corresponding unique feeling. We subjectively experience these phenomenal properties as properties of pain and it seems, in principle, possible that the corresponding dimensions form a domain onto which all instantiations of pain can be mapped. Thus, the sensory, affective, and evaluative properties seem crucial to better understand the nature of pain. It might be argued that this applies even if the considered phenomenal properties failed to satisfy the criterion of necessity in some rare exceptional cases. As we shall see in more detail in the following, the phenomenal variation of pains across sensory, affective, and evaluative dimensions provides a reliable guide in pain research, irrespective of whether pain constitutes a primitive phenomenal kind or not.

Generally speaking, the previous findings indicate that researchers should consider the complexity of the phenomenology of pains instead of merely focusing on some of their phenomenal properties. Otherwise we risk neglecting important information about the subject matter. Moreover, in scientific terms, we should take the findings outlined above seriously, explore the topology of the phenomenal space, and thereby fully exploit the potential of a multidimensional framework in reference to the spatially indicated similarities and dissimilarities of pains and non-pains alike. The usefulness of such an approach shall be highlighted within two fields of application. $^{6}$

First, the study of those brain processes involved in pain is crucial. Based on current knowledge, it does not seem possible to identify a specific brain area, neural network, or pattern of central activity for pain (Roy and Wager 2017). For this very reason, however, it might be useful to make connections between the phenomenal space and a "neural space" (e.g., Balkenius and Gärdenfors 2016; Kostic 2012; Price and Aydede 2005). We might also consider a bi-lateral method, using insights concerning one space to inform the construction of the other (Fink 2012). Even if no perfect isomorphism is evident, this linking can improve our understanding of the relationship between phenomenal and neuronal characteristics and, thus, motivate new approaches concerning, for instance, psychopharmacological or electrophysiological interventions to alter phenomenal aspects of pains. Interestingly, in the investigation of the correspondence between the phenomenal

\footnotetext{
${ }^{6}$ It is important to acknowledge that not all aspects of pain are best conceptualized in terms of quantifiable properties. For example, we must also make room for the more existential and holistic manner in which pain affects the life of patients (e.g., Kauppinen, 2020). Such aspects might be quantifiable in terms of their strength, but this hardly captures all relevant facets of an individual's lived experience. Thus, the multidimensional framework may constitute merely one building block in a comprehensive approach to pain and its treatment.
} 
space and a neural, it is not necessarily the relation between instantiations of pain but between instantiations of a more general class of homeostatic sensations that might prove to be of practical relevance (Coninx 2020a). For example, similarities between pains and non-pains might, in some cases, constitute a more beneficial guide than the similarities between pains occupying distant coordinate points in the phenomenal space.

Second, the construction of a phenomenal space constitutes a precondition for discussion of whether we can find evidence for an isomorphic relation of the phenomenal space with the "physical space" (Gärdenfors 2000) representing the properties of a mind-external object. The current state of science indicates that there is no distinct physical state that can be associated with all pains (Coninx 2021; Corns 2014a; Klein 2015). However, this does not exclude the usefulness of a phenomenal space. Taxonomies of phenomenal properties have been developed for the classification of different kinds of pain and their association with particular medical issues (Dubuisson and Melzack 1976; Melzack and Katz 2013). For instance, phenomenal qualifications constitute crucial instruments in the selection and evaluation of the effectiveness of clinical interventions (Baetu 2020). This general approach can be further pursued while rejecting the idea that pain can be reduced to a combination of phenomenal properties. Phenomenal spaces might function as diagnostic instruments, assuming that reports on certain phenomenal properties can be reliably correlated with numerous medical conditions and used as a guide to the treatments to be most likely successful. This is true even in the absence of a perfect isomorphism.

Even if the primitiveness of pain does not affect the scientific utility of investigating sensory, affective, and evaluative properties, one may still point out the mysterious character of the phenomenal quality which should be common and unique for pains but cannot be analyzed in terms of these phenomenal properties or their combination. To many philosophers, it seems uncontroversial that there is something that allows us to identify pains as pains from a first-person perspective. To others, it remains questionable whether our subjective experience reveals a phenomenal quality distinct from sensory, affective, and evaluative properties (e.g., Corns 2020). The general issue thereby is that proponents of both positions primarily rely on intuition and introspection and, as such, it may seem difficult to ever settle the dispute on the reality of the phenomenal quality in question (Aydede and Fulkerson 2019). In addition, many questions remain to be addressed even if we, for example, accept the existence of a fundamental "ouch" (e.g., Coninx 2020b).

To make a final decision on the question whether pain constitutes a phenomenal kind clearly exceeds the scope of this paper. I would just like to indicate that whether the primitiveness of pain proves problematic, in philosophical terms, depends on the precise understanding of the phenomenal kind status of pain. Philosophers commonly assume that there exists a phenomenal quality that is in fact common to all and only pains, that we can become introspectively aware of, and that, depending on the theory defended, can even exist without being subjectively accessible (e.g., Coleman 2020). However, we may only need a less demanding interpretation accepting that it typically feels to us like there is something common and distinct about pains, without further metaphysical commitments. Thus, the relevant question is rather 
why we subjectively experience pains this way. Again, the topology of the phenomenal space might prove to be a useful guide in future investigations.

\section{Conclusion}

The key findings of the paper can be summarized in three points. First, pain is a complex and variable phenomenon whose paradigmatic phenomenal properties can be modeled in a geometric space of sensory, affective, and evaluative dimensions. Second, it seems possible to map all kinds of pains onto coordinate points of this space, which thus might constitute an independent domain with integral dimensions. However, pain is not the only phenomenon that occupies coordinate points in the space. There is no phenomenal space formed of dimensions along which exclusively pains vary. Thus, pain seems to be a primitive phenomenal kind. Three, this does not mean that we have to reject the spatial framework as scientifically useless. On the contrary, exploring the topological structure of such space could be a crucial step in pain research. Similarly, philosophical consequences, for example concerning the primitiveness of pain, do not necessarily have to prove as striking as they first appear. Further consideration is needed to better understand the phenomenal kind status of pain.

Acknowledgements Many thanks to Sascha B. Fink, Lena Kästner, Colin Klein, Matej Kohár, Beate Krickel, Michelle Liu, Judith Martens, Albert Newen, Matías Osta Vélez, Luke Roelofs, Alfredo Vernazzani, Elmarie Venter, Pascale Willemsen, and Julia Wolf for inspiring discussions and thoughtful comments. I would also like to thank the anonymous reviewers for their helpful feedback.

Gefördert durch die Deutsche Forschungsgemeinschaft (DFG)—Projektnummer GRK-2185/1 (DFGGraduiertenkolleg Situated Cognition); Funded by the Deutsche Forschungsgemeinschaft (DFG, German Research Foundation)_-project number GRK-2185/1 (DFG Research Training Group Situated Cognition).

Funding Open Access funding enabled and organized by Projekt DEAL.

Open Access This article is licensed under a Creative Commons Attribution 4.0 International License, which permits use, sharing, adaptation, distribution and reproduction in any medium or format, as long as you give appropriate credit to the original author(s) and the source, provide a link to the Creative Commons licence, and indicate if changes were made. The images or other third party material in this article are included in the article's Creative Commons licence, unless indicated otherwise in a credit line to the material. If material is not included in the article's Creative Commons licence and your intended use is not permitted by statutory regulation or exceeds the permitted use, you will need to obtain permission directly from the copyright holder. To view a copy of this licence, visit http://creativecommons.org/licen ses/by/4.0/.

\section{References}

Aydede, M. (2005). Introduction: A critical and quasi-historical essay on theories of pain. In M. Aydede (Ed.), Pain: New essays on its nature and the methodology of its study (pp. 1-58). Cambridge: MIT Press.

Aydede, M. (2019). Does the IASP definition of pain need updating? Pain Reports, 4(5), e777. 
Aydede, M., \& Fulkerson, M. (2019). Reasons and theories of sensory affect. In D. Bain, M. Brady, \& J. Corns (Eds.), Philosophy of pain: Unpleasantness, emotion, and deviance (pp. 27-59). New York: Routledge.

Baetu, T. M. (2020). Pain in psychology, biology and medicine: some implications for pain eliminativism. Studies in History and Philosophy of Biological and Biomedical Sciences, 82, 101292.

Bain, D. (2013). What makes pains unpleasant? Philosophical Studies, 166(1), 69-89.

Bain, D. (2014). Pains that don't hurt. Australasian Journal of Philosophy, 92(2), 305-320.

Balkenius, C., \& Gärdenfors, P. (2016). Spaces in the brain: From neurons to meanings. Frontiers in Psychology, 7, 1820.

Barber, T. X. (1959). Towards a theory of pain: Relief of chronic pain by prefrontal leuocotomy, opiate, placebos, and hypnosis. Psychological Bulletin, 56(6), 430-460.

Berthier, M., Starkstein, S., \& Leiguarda, R. (1988). Asymbolia for pain: A sensory-limbic disconnection syndrome. Annals of Neurology, 24(1), 41-49.

Borg, E., Harrison, R., Stazicker, J., \& Salomons, T. (2020). Is the folk concept of pain polyeidic? Mind and Language, 35(1), 29-47.

Byrne, A. (2001). Intentionalism defended. Philosophical Review, 110(2), 199-240.

Clark, A. (2000). A theory of sentience. New York: Oxford University Press.

Clark, A. (2005). Painfulness is not a quale. In M. Aydede (Ed.), Pain: New essays on its nature and the methodology of its study (pp. 177-197). Cambridge: MIT Press.

Coleman, S. (2020). Painfulness, suffering, and consciousness. In D. Bain, M. Brady, \& J. Corns (Eds.), Philosophy of suffering: Metaphysics, value, and normativity (pp. 55-74). London: Routledge.

Coninx, S. (2020a). Experiencing pain: A scientific enigma and its philosophical solution. Berlin; New York: de Gruyter.

Coninx, S. (2020b). Pain, amnesia, and qualitative memory: Conceptual and empirical challenges. Journal of Consciousness Studies, 27(11-12), 126-133.

Coninx, S. (2021). Strong representationalism and bodily sensations: Reliable causal covariance and biological function. Philosophical Psychology, 34(2), 210-232.

Corns, J. (2014a). The inadequacy of unitary characterizations of pain. Philosophical Studies, 169(3), $355-378$.

Corns, J. (2014b). Unpleasantness, motivational oomph, and painfulness. Mind and Language, 29(2), 238-254.

Corns, J. (2018). Recent work on pain. Analysis, 78(4), 737-753.

Corns, J. (2020). The complex reality of pain. New York: Routledge.

Cutter, B. (2017). Pain and representation. In J. Corns (Ed.), Routledge handbook of philosophy of pain (pp. 29-39). Abingdon: Routledge.

Davis, K. D., Kucyi, A., \& Moayedi, M. (2015). The pain switch: an "ouch" detector. Pain, 156(11), 2164-2166.

Dubuisson, D., \& Melzack, R. (1976). Classification of clinical pain descriptions by multiple group discriminant analysis. Experimental Neurology, 51(2), 480-487.

Fields, H. L. (1999). Pain: An unpleasant topic. Pain, Supplement, 6, 61-69.

Fink, S. B. (2011). Independence and connections of pain and suffering. Journal of Consciousness Studies, 18(9-10), 46-66.

Fink, S. B. (2012). A natural state without a nature: Dealing with the ambiguity of pain in science and ethics. In H. McKenzie, J. Quintner, \& G. Bendelow (Eds.), At the edge of being: The aporia of pain (pp. 3-18). Oxford: Inter-Disciplinary Press.

Funkhouser, E. (2006). The determinable-determinate relation. Nous, 40(3), 548-569.

Gärdenfors, P. (2000). Conceptual spaces: The geometry of thought. Cambridge: MIT Press.

Gärdenfors, P., \& Warglien, M. (2012). Using conceptual spaces to model actions and events. Journal of Semantics, 29(4), 487-519.

Gärdenfors, P., Jost, J., \& Warglien, M. (2018). From actions to effects: Three constraints on event mappings. Frontiers in Psychology, 9, 1391.

Grahek, N. (2007). Feeling pain and being in pain (2nd ed.). Cambridge: MIT Press.

Hall, R. J. (1989). Are pains necessarily unpleasant? Philosophy and Phenomenological Research, 49, 643-659.

Kauppinen, A. (2020). The world according to suffering. In D. Bain, M. Brady, \& J. Corns (Eds.), Philosophy of suffering: metaphysics, value, and normativity (pp. 19-36). London: Routledge.

Keats, A. S., \& Beecher, H. K. (1950). Pain relief with hypnotic doses of barbiturates and a hypothesis. The Journal of Pharmacology and Experimental Therapeutics, 100(1), 1-13. 
Klein, C. (2015). What the body commands - the imperative theory of pain. Cambridge: MIT Press.

Kostic, D. (2012). The vagueness constraint and the quality space for pain. Philosophical Psychology, 25(6), 929-939.

Kripke, S. A. (1981). Naming and necessity. Oxford: Blackwell Publishing.

McClelland, T. (2020). The disruption model of suffering. In D. Bain, M. Brady, \& J. Corns (Eds.), Philosophy of suffering: metaphysics, value, and normativity (pp. 37-54). Abingdon: Routledge.

Melzack, R. (1961). The perception of pain. Scientific American, 204(2), 41-49.

Melzack, R. (2005). The McGill Pain Questionnaire: From description to measurement. Anesthesiology, 103(1), 199-202.

Melzack, R., \& Wall, P. D. (1982). The challenge of pain (2nd ed.). London: Penguin Books.

Melzack, R., \& Katz, J. (2013). Pain measurement in adult patients. In S. B. McMahon, M. Koltzenburg, I. Tracey, \& D. Turk (Eds.), Wall and Melzack's textbook of pain (6th ed., pp. 301-314). Philadelphia: Elsevier.

Osta-Vélez, M., \& Gärdenfors, P. (2020). Category-based induction in conceptual spaces. Journal of Mathematical Psychology, 96, 102357.

Paice, J. A., Penn, R. D., \& Shott, S. (1996). Intraspinal morphine for chronic pain: A retrospective, multicenter study. Journal of Pain and Symptom Management, 11(2), 71-80.

Park, T. (2019). On the alleged evidence for non-unpleasant pains. Inquiry. https://doi.org/10.1080/00201 74X.2019.1658625.

Ploner, M., Freund, H. J., \& Schnitzler, A. (1999). Pain affect without pain sensation in a patient with a postcentral lesion. Pain, 81(1-2), 211-214.

Price, D. D. (2000). Psychological and neural mechanisms of the affective dimension of pain. Science, 288(5472), 1769-1772.

Price, D. D., \& Aydede, M. (2005). The experimental use of introspection in the scientific study of pain and its integration with third-person methodologies: The experiental-phenomenological approach. In M. Aydede (Ed.), Pain: new essays on its nature and the methodology of its study (pp. 243-273). Massachusetts: MIT Press.

Raja, S. N., Carr, D. B., Cohen, M., Finnerup, N. B., Flor, H., Gibson, S., et al. (2020). The revised International Association for the Study of Pain definition of pain: concepts, challenges, and compromises. Pain. https://doi.org/10.1097/j.pain.0000000000001939.

Roy, M., \& Wager, T. D. (2017). Neuromatrix theory of pain. In J. Corns (Ed.), Routledge handbook of philosophy of pain (pp. 87-97). New York: Routledge.

Wierzbicka, A. (2012). Is pain a human universal? A cross-linguistic and cross-cultural perspective on pain. Emotion Review, 4(3), 307-317.

Young, B. D., Keller, A., \& Rosenthal, D. (2014). Quality-space theory in olfaction. Frontiers in Psychology, 5(1), 116-130.

Publisher's Note Springer Nature remains neutral with regard to jurisdictional claims in published maps and institutional affiliations. 